(2) Open Access Full Text Article

\title{
Drug-induced tubulointerstitial nephritis in a retrospective study using spontaneous reporting system database
}

This article was published in the following Dove Press journal:

Therapeutics and Clinical Risk Management

\section{Saki Oyama \\ Keiko Hosohata \\ Ayaka Inada \\ Iku Niinomi \\ Yasuhiro Mori \\ Yuki Yamaguchi \\ Mayako Uchida \\ Kazunori Iwanaga}

Education and Research Center for Clinical Pharmacy, Osaka University of Pharmaceutical Sciences, Takatsuki, Osaka, Japan
Correspondence: Keiko Hosohata Education and Research Center for Clinical Pharmacy, Osaka University of Pharmaceutical Sciences, 4-20-I Nasahara, Takatsuki, Osaka 569-1094, Japan

Tel $+8|72690| 27 \mid$

Fax +81726901023

Email hosohata@gly.oups.ac.jp
Introduction: Tubulointerstitial nephritis (TIN) is a problem in clinical settings because drug therapy is the cause in most cases. Patients often present with nonspecific symptoms, which can lead to delays in the diagnosis and treatment of the disease. The purpose of this study was to clarify the rank-order of the association of TIN with the causative drugs using a spontaneous reporting system database.

Materials and methods: Data were extracted from the Japanese Adverse Drug Event Report database of the Pharmaceuticals and Medical Devices Agency (Japan). Based on 5,195,890 reports of all adverse reactions, we obtained 3,088 reports of TIN caused by all drugs and calculated the reporting odds ratio (ROR) and 95\% CI for TIN.

Results: The 5 drugs with the highest RORs were gliclazide (ROR, 30.5; 95\% CI, 17.4-53.2), tosufloxacin tosilate hydrate (ROR, 29.5; 95\% CI, 21.3-41.0), piperacillin-tazobactam (ROR, 24.3; 95\% CI, 19.4-30.5), cefteram pivoxil (ROR, 23.5; 95\% CI, 12.5-44.2), and mefenamic acid (ROR, 22.5; 95\% CI, 13.4-37.7). No sex-related difference was observed in drug-induced TIN. Most of the reports about TIN onset following the administration of culprit drugs were recorded within 12 weeks.

Conclusion: Based on the results, a comprehensive study using a pharmacovigilance database enabled us to identify the dugs that most frequently induced TIN, so these drugs should be used carefully in clinical practice to avoid TIN.

Keywords: tubulointerstitial nephritis, pharmacovigilance, spontaneous reporting system, reporting odds ratio, Japanese Adverse Drug Event Report database, JADER

\section{Introduction}

There has been an increasing incidence of tubulointerstitial nephritis (TIN). ${ }^{1,2}$ TIN is characterized by histologic interstitial abnormalities that reflect infiltration by various inflammatory cells, including lymphocytes, plasma cells, and macrophages, ${ }^{3}$ and is often accompanied by tubulitis. The common clinical presentations are rash, fever, eosinophilia, and elevated immunoglobulin E ( $\operatorname{IgE}$ ) levels, but patients often present with nonspecific symptoms, which can lead to delayed diagnosis and treatment of the disease, resulting in renal dysfunction. ${ }^{4}$

The causes of TIN vary and include drug reactions, ${ }^{5}$ infections,${ }^{6}$ and autoimmune diseases $^{7}$ such as Sjögren syndrome or IgG4-related disease. Recently, drug-induced TIN has accounted for more than two-thirds of the cases. ${ }^{8}$ A large number of drugs have been associated with TIN, and any drug can theoretically induce an episode of acute TIN. In most patients, renal function improves after discontinuation of the drug, but the renal function does not recover fully in some cases. ${ }^{9}$ TIN often occurs without 
any abnormal urinalysis findings, which makes it difficult to identify at an early stage. ${ }^{10}$ Therefore, it is important to know which drugs more often cause TIN in clinical settings. However, few studies have performed surveillance of the rank ordering of the drugs associated with TIN. The objective of this study was to conduct a comprehensive nationwide overview of drug-induced TIN using a spontaneously reported adverse drug reactions (ADRs) database in Japan.

\section{Materials and methods}

The present study used data from the public release of Pharmaceuticals and Medical Devices Agency's Japanese Adverse Drug Event Report (JADER) database, which contains information on the ADRs and patients in Japan since April 1, 2004. We used data from the JADER to which adverse event reports were submitted between April 2004 and January 2017. The data structure of JADER consists of 4 data sets: patient demographic information (DEMO), drug information (DRUG), adverse reactions (REAC), and medical history. In REAC table, the Medical Dictionary for Regulatory Activities (MedDRA) is used to codify the adverse reactions, which are indicated as "Preferred Term (PT)".

After we removed duplicated data from each table, ${ }^{11}$ the DEMO table was then linked to the REAC and DRUG tables using the ID number. In each case, the contribution of the medication to adverse reactions was classified into three categories: "suspected medicine," "concomitant medicine," and "interaction." A "suspected medicine" is defined as a pharmaceutical product with which an adverse reaction is suspected to be associated. When the reporter suspects an interaction, he/she reports it as an "interaction." A "concomitant medicine" is defined as another pharmaceutical product used at the time of the adverse reaction.

We only extracted cases that were classified as "suspected medicine" and analyzed the reports of suspected drugs and ADRs, which we selected as "TIN" in the PT coded in MedDRA (version 20.1). We compiled a crosstabulation table based on two classifications: the presence or absence of TIN and the presence or absence of the suspected medicine. Therefore, we calculated the reporting odds ratio (ROR). The ROR is rate of reporting a specific adverse reaction caused by a particular drug divided by the rate of the same adverse reactions caused by all other drugs present in the database. In addition, the ROR was frequently used with the spontaneous reporting database as an index of the relative risk of drug-associated adverse reactions. A signal was considered to be present when the lower limit of the $95 \%$ CI of the ROR was $>1$.
In this database, age, height, and weight information are indicated in the form of age in decades, height in centimeterdenominated ranges, and weight in kilogram-denominated ranges. Because these data are not continuous variables, we could not conduct multiple analyses using them. All analyses were performed with JMP Pro 12 (SAS Institute Inc., Cary, NC, USA).

\section{Results}

In total, 5,195,890 reports were obtained after the combination of three tables, DRUG $(2,850,470$ reports), REAC (709,826 reports), and DEMO (449,558 patients), with the ID number. Of those, we extracted suspected drugs causing all adverse reactions (1,984,122 reports) and obtained 3,088 reports of suspected drugs and TIN (corresponding to $0.16 \%$ of all records in the JADER). The annual numbers of reports for 2004-2016 are shown in Table 1. Of note, the number of deaths associated with TIN was extremely low, but the rate of nonrecovery from drug-induced TIN was high. As shown in Table 2, approximately $60 \%$ of the patients were men. According to the age distribution of the study population, TIN occurred frequently in those in their 60s $(21.6 \%)$. The most frequent duration of treatment until TIN was within 1 month (16.8\%) (1 week, 12.0\%; 2 weeks, 3.6\%; 4 weeks, $1.2 \%$ ), and the second most frequent was 12 weeks $(13.7 \%)$. The TIN outcomes were distributed as follows: $0.3 \%$ of the patients died, $65.8 \%$ had recovered or were recovering, $12.1 \%$ had not recovered, 5.6\% had after-effects, and the outcome was unknown in the remaining $16.2 \%$.

In our analysis, 427 different drugs were "suspected" in cases of TIN. Of these, the drugs ranked in order of the frequency of a reported association with TIN were examined. The first 65 medications gave a positive signal, that is, the lower confidence limit of the ROR was $>1$ (Table 3). The most frequently reported drug was gliclazide (ROR, 30.5; 95\% CI, 17.4-53.2), followed by tosufloxacin tosilate hydrate (ROR, 29.5; 95\% CI, 21.3-41.0), piperacillintazobactam (ROR, 24.3; 95\% CI, 19.4-30.5), cefteram pivoxil (ROR, 23.5; 95\% CI, 12.5-44.2), mefenamic acid (ROR, 22.5; 95\% CI, 13.4-37.7), cefotaxime sodium (ROR, 21.5; 95\% CI, 11.5-40.5), and mesalazine (ROR, 20.1; $95 \% \mathrm{CI}, 16.1-25.0)$.

\section{Discussion}

TIN is a frequent and important clinical problem that can be induced by a variety of medications. In our results, of the $1,984,122$ reports of ADRs recorded in JADER during the study period, $3,088(0.16 \%)$ corresponded to TIN. Our results 


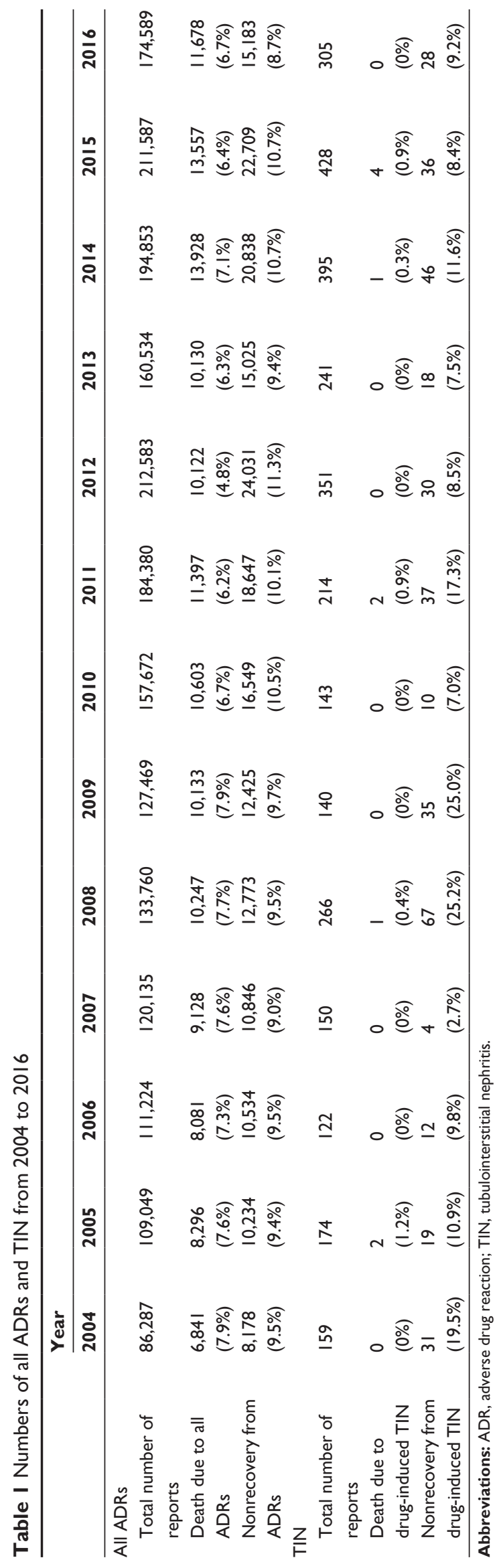

Table 2 Characteristics of the patients with drug-induced TIN

\begin{tabular}{lll}
\hline Variables & $\mathbf{N}$ & Percent \\
\hline Sex & & \\
Men & 1,766 & 57.2 \\
Women & $1,30 \mathrm{I}$ & 42.1 \\
Unknown & 21 & 0.7 \\
Age (years) & & \\
Under I0 & 137 & 4.5 \\
I0s & 270 & 8.7 \\
20s & 153 & 5.0 \\
30s & 239 & 7.7 \\
40 s & 271 & 8.8 \\
50 s & 415 & 13.4 \\
60 s & 668 & 21.6 \\
70 s & 510 & 16.5 \\
80 s & 335 & 10.9 \\
90 s & 37 & 1.2 \\
Unknown & 53 & 1.7 \\
Period for treatment at the episode & \\
I week & 371 & 12.0 \\
2 weeks & 110 & 3.6 \\
4 weeks & 36 & 1.2 \\
I2 weeks & 422 & 13.7 \\
24 weeks & 63 & 2.0 \\
I year & 212 & 6.9 \\
After I year & 211 & 6.8 \\
Unknown & 1,663 & 53.8 \\
\hline A & &
\end{tabular}

Abbreviation: TIN, tubulointerstitial nephritis.

also reveal that the duration of the exposure to drugs until the onset of TIN was most often within 12 weeks. Furthermore, we found that most TIN survivors achieved recovery or remission after the index TIN episode. However, the recovery from TIN was worse compared to all other ADRs across the 12-year time span.

The clinical presentation of TIN is highly variable. The only consistent clinical manifestation is acute or subacute kidney injury, often resulting in chronic kidney disease (CKD). ${ }^{12,13}$ The most common etiology of TIN is drug-induced disease, which is thought to underlie approximately $70 \%$ of cases. ${ }^{8}$ Antibiotics,${ }^{14}$ antiviral medications, ${ }^{15}$ anticonvulsants, ${ }^{16}$ analgesics,${ }^{17}$ and gastrointestinal medications $^{18}$ are associated with TIN. Historically, the prevalence of TIN was reported to be higher in patients over 60 years than in those under 60 years. ${ }^{19}$ A Spanish biopsy registry study from 1994 to 2009 showed that the prevalence of TIN had markedly increased in elderly patients. ${ }^{1}$ Consistent with these reports, our results reveal that many of the patients with TIN were elderly (most frequently in their 60s). It has been postulated that the reason may be because of a reduced kidney function, which results in the accumulation of drugs and/or their metabolites, resulting in an increased risk of developing serious side effects. Another possibility is that 
Table 3 Most frequently reported drugs that induce TIN

\begin{tabular}{|c|c|c|c|}
\hline Suspected drug & $\begin{array}{l}\text { Cases } \\
\text { (n) }\end{array}$ & ROR & $95 \% \mathrm{Cl}$ \\
\hline Gliclazide & 13 & 30.5 & $17.4-53.2$ \\
\hline Tosufloxacin tosilate hydrate & 38 & 29.5 & $21.3-41.0$ \\
\hline Piperacillin-tazobactam & 80 & 24.3 & $19.4-30.5$ \\
\hline Cefteram pivoxil & 10 & 23.5 & $12.5-44.2$ \\
\hline Mefenamic acid & 15 & 22.5 & 13.4-37.7 \\
\hline Cefotaxime sodium & 10 & 21.5 & $11.5-40.5$ \\
\hline Mesalazine & 85 & 20.1 & $16.1-25.0$ \\
\hline Piperacillin sodium & 97 & 19.6 & $|6.0-24|$. \\
\hline Polaprezinc & 10 & 19.3 & $10.3-36.2$ \\
\hline Teneligliptin hydrobromide hydrate & II & 17.8 & $9.8-32.4$ \\
\hline Benidipine hydrochloride & 14 & 17.4 & $10.2-29.6$ \\
\hline Loxoprofen sodium hydrate & 205 & 15.8 & $13.7-18.2$ \\
\hline Ketoprofen & 11 & 15.5 & $8.5-28.3$ \\
\hline Loratadine & 10 & 14.4 & $7.7-27.0$ \\
\hline Cefdinir & 21 & 14.1 & $9.2-21.8$ \\
\hline Tretinoin & 12 & 13.6 & $7.7-24.2$ \\
\hline Cefditoren pivoxil & 23 & 12.8 & $8.5-19.4$ \\
\hline Ibuprofen & 14 & 12.7 & $7.5-21.5$ \\
\hline Ursodeoxycholic acid & 17 & 11.6 & $7.2-18.8$ \\
\hline Pyrazinamide & 17 & 11.6 & $7.2-18.8$ \\
\hline Acetaminophen & 116 & 11.5 & $9.6-13.9$ \\
\hline Limaprost alfadex & 13 & 11.5 & $6.7-20.0$ \\
\hline Atazanavir sulfate & 11 & 10.3 & $5.7-18.8$ \\
\hline Omeprazole & 39 & 10.2 & $7.4-14.1$ \\
\hline Rebamipide & 43 & 10.2 & $7.5-13.8$ \\
\hline Enalapril maleate & 20 & 10.2 & $6.5-15.9$ \\
\hline Rifampicin & 35 & 9.6 & $6.9-13.5$ \\
\hline Cefcapene pivoxil hydrochloride hydrate & 33 & 9.4 & $6.6-13.2$ \\
\hline Montelukast sodium & 10 & 8.3 & $4.4-15.5$ \\
\hline Abacavir sulfate & 14 & 7.2 & $4.2-12.1$ \\
\hline Eldecalcitol & 14 & 7.1 & $4.2-12.0$ \\
\hline Vancomycin hydrochloride & 40 & 7.1 & $5.2-9.7$ \\
\hline Ethambutol hydrochloride & 20 & 6.9 & $4.4-10.7$ \\
\hline Moxifloxacin hydrochloride & 10 & 6.7 & $3.6-12.6$ \\
\hline Levofloxacin hydrate & 58 & 6.7 & $5.1-8.7$ \\
\hline Diclofenac sodium & 66 & 6.6 & $5.2-8.5$ \\
\hline Famotidine & 50 & 6.2 & $4.7-8.2$ \\
\hline Isoniazid & 21 & 5.5 & $3.6-8.5$ \\
\hline Candesartan cilexetil & 24 & 5.2 & $3.5-7.7$ \\
\hline Minocycline hydrochloride & 18 & 5.1 & $3.2-8.1$ \\
\hline Risedronate sodium hydrate & 13 & 5.1 & $2.9-8.8$ \\
\hline Clarithromycin & 40 & 5.0 & $3.6-6.8$ \\
\hline Ceftriaxone sodium hydrate & 27 & 4.9 & $3.4-7.2$ \\
\hline Cefazolin sodium & 15 & 4.6 & $2.8-7.7$ \\
\hline Lansoprazole & 45 & 4.5 & $3.3-6.0$ \\
\hline Allopurinol & 42 & 4.4 & $3.2-5.9$ \\
\hline Rosuvastatin calcium & 14 & 4.2 & $2.5-7.2$ \\
\hline Sodium rabeprazole & 13 & 4.1 & $2.4-7.2$ \\
\hline Ampicillin-sulbactam & 11 & 4.1 & $2.3-7.5$ \\
\hline Meropenem hydrate & 22 & 4.1 & $2.7-6.3$ \\
\hline Carbocisteine & 12 & 4.0 & $2.3-7.1$ \\
\hline Amoxicillin hydrate & 25 & 3.9 & $2.7-5.9$ \\
\hline Adefovir pivoxil & 11 & 3.9 & $2.2-7.1$ \\
\hline Garenoxacin mesilate hydrate & 15 & 3.8 & $2.3-6.3$ \\
\hline Atorvastatin calcium hydrate & 18 & 3.1 & $1.9-4.9$ \\
\hline Lamivudine & 18 & 2.9 & $1.8-4.7$ \\
\hline
\end{tabular}

Table 3 (Continued)

\begin{tabular}{llll}
\hline Suspected drug & $\begin{array}{l}\text { Cases } \\
\text { (n) }\end{array}$ & ROR & 95\% CI \\
\hline Glimepiride & 15 & 2.9 & I.7-4.8 \\
Amlodipine besilate & 25 & 2.9 & $\mathrm{I} .9-4.3$ \\
Sodium valproate & 30 & 2.6 & $\mathrm{I} .8-3.8$ \\
Aspirin & 32 & 2.3 & $\mathrm{I} .7-3.3$ \\
Trimethoprim-Sulfamethoxazole & 17 & 2.2 & $\mathrm{I} .3-3.5$ \\
Celecoxib & 12 & 1.9 & $\mathrm{I} . \mathrm{I}-3.3$ \\
Valaciclovir hydrochloride & $\mathrm{I} 7$ & $\mathrm{I} .7$ & $\mathrm{I} . \mathrm{I}-2.7$ \\
Pregabalin & 27 & $\mathrm{I} .7$ & $\mathrm{I} .2-2.5$ \\
Carbamazepine & 29 & $\mathrm{I} .5$ & $\mathrm{I} . \mathrm{I}-2.2$ \\
\hline
\end{tabular}

Abbreviations: ROR, reporting odds ratio; TIN, tubulointerstitial nephritis.

the elderly have concomitant diseases, such as cardiovascular disease, CKD, diabetes, or heart failure; therefore, they have higher rates of diagnostic procedures and take several nephrotoxic agents. ${ }^{20}$

We found the duration of exposure to drugs until TIN onset was most often within 12 weeks. This finding is line with a single-center retrospective analysis showing that 8 out of 14 cases with presumed drug-related TIN could be attributed to proton pump inhibitors (PPIs) and that the duration of PPI treatment before presentation was between 2 weeks and 9 months. ${ }^{21}$

Of note, our results revealed that gliclazide, a sulphonylurea-class molecule used to control glycemic levels in patients with noninsulin-dependent diabetes mellitus, exhibited the highest ROR of TIN. So far, there have been few studies on the renal toxicity of gliclazide. In addition, the Japanese medical package insert of gliclazide (Glimicron ${ }^{\circledR}$; Sumitomo Dainippon Pharma Co., Ltd., Osaka, Japan) state that the frequency of ADRs affecting the kidney is very low (increases in blood urea nitrogen and serum creatinine are under $0.1 \%$ and unknown, respectively). To the best of our knowledge, this is the first study to report the association of gliclazide with TIN.

In our results, antibiotics (such as tosufloxacin tosilate hydrate, piperacillin-tazobactam, cefteram pivoxil, and cefotaxime sodium), nonsteroidal anti-inflammatory drugs (NSAIDs) (such as mefenamic acid, loxoprofen sodium hydrate, and ketoprofen), and drugs against methicillinresistant Staphylococcus aureus (MRSA) (such as vancomycin and rifampicin) exhibited high RORs. This observation is in accordance with previous reports showing that the main causes of TIN were antibiotics, NSAIDs, and anti-MRSA antibiotics. ${ }^{1,9,22-24}$ Recently, PPI-induced TIN has been increasingly reported. Biopsy-proven TIN from 1993 to 2011 showed that drug-induced TIN was due to antibiotics in $49 \%$ of the cases, PPIs in $14 \%$, and NSAIDs in $11 \% .{ }^{10} \mathrm{In}$ 
the present study, we also found that the ROR of omeprazole was significantly higher.

Importantly, we found that mesalazine-induced TIN was frequently reported. There have been few reports on the outcome of mesalazine-induced TIN. A case report showed that the withdrawal of mesalazine and initiation of intravenous methylprednisolone led to the recovery of a patient's renal function. ${ }^{25}$ However, our results revealed that there were high frequencies of nonrecovering mesalazine-induced TIN.

\section{Limitations}

The JADER database is considered to be a valuable tool; ${ }^{26}$ however, several limitations inherent to spontaneous reporting are included. First, the JADER database has various biases, such as the lack of a denominator that indicates the total number of patients who received the drugs of interest, as well as missing data and confounding factors. Second, the ROR does not provide a robust indication of the signal strength. In spontaneous reporting systems such as JADER, control populations are not included, so the ROR is different from the "odds ratio" that is commonly used in epidemiological studies. In real terms, the ROR indicates an increased risk of adverse event reporting, and not the risk of adverse reactions. Third, the date of outcome is not included in this database. Therefore, it is difficult to assess the average duration between stopping the offending drug and recovery of renal function. Finally, the present method did not provide us with detailed information on the patients' clinical status. Clinically unstable patients are more likely to develop TIN and to be taking several concomitant drugs than stable patients; this situation may be a confounding factor when estimating the occurrence of TIN.

\section{Conclusion}

The suspected drugs associated with TIN determined using a nationwide pharmacovigilance database strongly suggest that physicians should be alerted to take precautions against drugs inducing TIN, select appropriate therapeutic medicine, and potentially avoid TIN.

\section{Acknowledgment}

This work was supported in part by the Science Research Promotion Fund.

\section{Disclosure}

The authors report no conflicts of interest in this work.

\section{References}

1. Goicoechea M, Rivera F, Lopez-Gomez JM; Spanish Registry of Glomerulonephritis. Increased prevalence of acute tubulointerstitial nephritis. Nephrol Dial Transplant. 2013;28(1):112-115.
2. Wilson GJ, Kark AL, Francis LP, Hoy W, Healy HG, Mallett AJ. The increasing rates of acute interstitial nephritis in Australia: a single centre case series. BMC Nephrol. 2017;18(1):329.

3. Colvin RB, Burton JR, Hyslop NE Jr, Spitz L, Lichtenstein NS. Letter: penicillin-associated interstitial nephritis. Ann Intern Med. 1974;81(3): 404-405.

4. Councilman WT. Acute interstitial nephritis. J Exp Med. 1898;3(4-5): 393-420.

5. Rossert J. Drug-induced acute interstitial nephritis. Kidney Int. 2001; 60(2):804-817.

6. Schwarz A, Krause PH, Kunzendorf U, Keller F, Distler A. The outcome of acute interstitial nephritis: risk factors for the transition from acute to chronic interstitial nephritis. Clin Nephrol. 2000;54(3):179-190.

7. Saeki T, Nishi S, Imai N, et al. Clinicopathological characteristics of patients with igg4-related tubulointerstitial nephritis. Kidney Int. 2010;78(10):1016-1023.

8. Baker RJ, Pusey CD. The changing profile of acute tubulointerstitial nephritis. Nephrol Dial Transplant. 2004;19(1):8-11.

9. Tanaka T, Nangaku M. Pathogenesis of tubular interstitial nephritis. Contrib Nephrol. 2011;169:297-310.

10. Muriithi AK, Leung N, Valeri AM, et al. Biopsy-proven acute interstitial nephritis, 1993-2011: a case series. Am J Kidney Dis. 2014;64(4): $558-566$.

11. Bate A, Evans SJ. Quantitative signal detection using spontaneous ADR reporting. Pharmacoepidemiol Drug Saf. 2009;18(6):427-436.

12. Michel DM, Kelly CJ. Acute interstitial nephritis. J Am Soc Nephrol. 1998;9(3):506-515.

13. Kodner CM, Kudrimoti A. Diagnosis and management of acute interstitial nephritis. Am Fam Physician. 2003;67(12):2527-2534.

14. Toto RD. Acute tubulointerstitial nephritis. Am J Med Sci. 1990; 299(6):392-410.

15. Perazella MA. Acute renal failure in HIV-infected patients: a brief review of common causes. Am J Med Sci. 2000;319(6):385-391.

16. Hoffman EW. Phenytoin-induced interstitial nephritis. South Med J. 1981;74(9):1160-1161.

17. Pirani CL, Valeri A, D’Agati V, Appel GB. Renal toxicity of nonsteroidal anti-inflammatory drugs. Contrib Nephrol. 1987;55:159-175.

18. Ruffenach SJ, Siskind MS, Lien YH. Acute interstitial nephritis due to omeprazole. Am J Med. 1992;93(4):472-473.

19. Davison AM, Jones CH. Acute interstitial nephritis in the elderly: A report from the UK MRC glomerulonephritis register and a review of the literature. Nephrol Dial Transplant. 1998;13(Suppl 7):12-16.

20. Zhang L, Fu P, Wang L, et al. The clinical features and outcome of crush patients with acute kidney injury after the Wenchuan earthquake: differences between elderly and younger adults. Injury. 2012;43(9): 1470-1475.

21. Torpey N, Barker T, Ross C. Drug-induced tubulo-interstitial nephritis secondary to proton pump inhibitors: experience from a single UK renal unit. Nephrol Dial Transplant. 2004;19(6):1441-1446.

22. Muriithi AK, Leung N, Valeri AM, et al. Clinical characteristics, causes and outcomes of acute interstitial nephritis in the elderly. Kidney Int. 2015;87(2):458-464.

23. Hsu SI. Biopsy-proved acute tubulointerstitial nephritis and toxic epidermal necrolysis associated with vancomycin. Pharmacotherapy. 2001;21(10):1233-1239.

24. Gabow PA, Lacher JW, Neff TA. Tubulointerstitial and glomerular nephritis associated with rifampin. Report of a case. JAMA. 1976; 235(23):2517-2518.

25. Tadic M, Grgurevic I, Scukanec-Spoljar M, et al. Acute interstitial nephritis due to mesalazine. Nephrology (Carlton). 2005;10(2):103-105.

26. Fujiwara M, Kawasaki Y, Yamada H. A pharmacovigilance approach for post-marketing in Japan using the Japanese Adverse Drug Event Report (JADER) database and association analysis. PLoS One. 2016; 11(4):e0154425. 
Therapeutics and Clinical Risk Management

Dovepress

\section{Publish your work in this journal}

Therapeutics and Clinical Risk Management is an international, peerreviewed journal of clinical therapeutics and risk management, focusing on concise rapid reporting of clinical studies in all therapeutic areas, outcomes, safety, and programs for the effective, safe, and sustained use of medicines. This journal is indexed on PubMed Central, CAS,

EMBase, Scopus and the Elsevier Bibliographic databases. The manuscript management system is completely online and includes a very quick and fair peer-review system, which is all easy to use. Visit http://www.dovepress.com/testimonials.php to read real quotes from published authors.

Submit your manuscript here: http://www.dovepress.com/therapeutics-and-clinical-risk-management-journal 